\title{
Declaraciones institucionales y percepciones individuales sobre la sustentabilidad en escuelas de negocios mexicanas ${ }^{\text {is }}$
}

\author{
Institutional statements and individual perceptions of sustainability \\ in Mexican business schools
}

\section{Gabriela Monforte García*, Andreas Michael Hartmann \\ y Gabriela María Farías Martínez \\ ITESM, Campus Monterrey, México}

Recibido el 5 de noviembre de 2015; aceptado el 27 de abril de 2016

Disponible en Internet el 17 de noviembre de 2016

\section{Resumen}

El objetivo de este trabajo es analizar cómo las escuelas de negocios en las universidades en México manejan el concepto de sustentabilidad como parte de su tarea educativa. Para lograr una visión integral se tomaron en cuenta los tres ejes de la sustentabilidad: el ambiental, el social y el económico. Después de discutir las dimensiones conceptuales de la sustentabilidad, este trabajo presenta dos secciones empíricas: primero un análisis de contenidos del concepto en documentos institucionales y planes de estudio de carreras profesionales en las áreas de los negocios, y luego una encuesta sobre la percepción del concepto de sustentabilidad que manifiestan profesores y alumnos de las instituciones evaluadas. Los análisis indican que profesores y estudiantes en las disciplinas contables y administrativas requieren reforzar la conciencia sobre los aspectos económico y ambiental de la sustentabilidad. Los resultados proporcionan información que permitirá dirigir los esfuerzos de las instituciones de educación superior por capacitar a
\end{abstract}

\footnotetext{
¿st En esta investigación los términos desarrollo sostenible y sustentable se consideran como sinónimos, asumiendo que es aquel que permite satisfacer las necesidades de las generaciones presentes sin comprometer la satisfacción de las necesidades de las generaciones futuras.

* Autor para correspondencia.

Correo electrónico: gmonforte@itesm.mx (G. Monforte García).

La revisión por pares es responsabilidad de la Universidad Nacional Autónoma de México. 
sus profesores así como ofrecer una formación integral a sus estudiantes que abarque los tres ejes de la sustentabilidad.

(C) 2016 Universidad Nacional Autónoma de México, Facultad de Contaduría y Administración. Este es un artículo Open Access bajo la licencia CC BY-NC-ND (http://creativecommons.org/licenses/by-nc-nd/4.0/).

Códigos JEL: A22; I21; I29

Palabras clave: Educación superior; Escuelas de negocios; Educación para el desarrollo sostenible

\begin{abstract}
The objective of this paper is to analyze how business schools of Mexican universities deal with the concept of sustainability as part of their educational task. In order to achieve a comprehensive vision, this study considers the environmental, the social, and the economic components of sustainability. After discussing the conceptual dimensions of education for sustainability, the empirical part of the paper consists of two sections: a content analysis of how official university documents handle the concept of sustainability and a survey of professors' and students' perceptions of sustainability. The analysis shows that both professors and students in the Management and Accounting disciplines need to extend their understanding about the economic and environmental aspects of sustainability. The results of this study provide information that can guide the efforts made by institutions of higher learning for training their professors and offering a comprehensive education for their students in all three components of sustainability.

(C) 2016 Universidad Nacional Autónoma de México, Facultad de Contaduría y Administración. This is an open access article under the CC BY-NC-ND license (http://creativecommons.org/licenses/by-nc-nd/4.0/).
\end{abstract}

JEL classification: A22; I21; I29

Keywords: Higher education; Business schools; Education for sustainability

\title{
Introducción
}

La educación ambiental tiene su origen en los años setenta. En la Conferencia de las Naciones Unidas sobre el Medio Ambiente Humano realizada en 1972 en Estocolmo, Suecia, se planteó la necesidad de corregir los problemas ambientales, principalmente los que afectaban a la salud humana, aunque sin buscar un cambio en los estilos de desarrollo (CECADESU, 2012). En 1975, en el Seminario Internacional de Educación Ambiental, celebrado en Belgrado, ya se consideraba prioritaria a la educación en esos procesos de cambio (CECADESU, 2012). A partir de ese momento «se concibe a la educación ambiental como herramienta que contribuya a la formación de una nueva ética universal que reconozca las relaciones del hombre con el hombre y con la naturaleza» (CECADESU, 2012, p. 7). Fue en 1977, hasta la Conferencia Intergubernamental de Tiblisi, cuando se planteó una educación ambiental diferente a la educación tradicional, donde «los principios rectores de la educación ambiental son la comprensión de las articulaciones económicas políticas y ecológicas de la sociedad y la necesidad de considerar al medio ambiente en su totalidad» (CECADESU, 2012, p. 7). Más de 10 años después, en la Cumbre de la Tierra celebrada en Río de Janeiro, Brasil, en 1972, a través de la Agenda21 se reorienta la educación ambiental hacia el desarrollo sostenible. En paralelo a la Cumbre de la Tierra se realizó el Foro Global Ciudadano de Río 92, donde se aprobó el Tratado de Educación Ambiental hacia Sociedades Sustentables y de Responsabilidad Global. En dicho tratado se señala a la educación para la sustentabilidad como un proceso de aprendizaje permanente basado en el respeto a todas las formas de vida, planteando la necesidad de revocar los programas que soportan el modelo de crecimiento económico que aún es vigente (CECADESU, 2012). 
Con el propósito de robustecer el avance en la educación para la sustentabilidad, se designó a la UNESCO como la coordinadora del Decenio de las Naciones Unidas de la Educación para el Desarrollo Sostenible (2005-2014), estableciendo que educar para la sustentabilidad significa «incorporar los temas fundamentales del desarrollo sostenible a la enseñanza y el aprendizaje. ..». Asimismo, se señala que este reto demanda la incorporación de «...métodos participativos de enseñanza y aprendizaje que motiven a los alumnos y les doten de autonomía, a fin de cambiar su conducta y facilitar la adopción de medidas en pro del desarrollo sostenible...» (UNESCO, s.f.,a, p. 1).

Lo anterior fue enfatizado en la declaración Aichi-Nagoya (UNESCO, 2014), la cual resalta la importancia de la educación para el desarrollo sustentable como medio para desarrollar una perspectiva de ciudadanía global dentro de un contexto de retos locales, presentes y futuros. Además, en la declaración de Incheon (UNESCO, 2015) se establece que la educación de calidad propicia el desarrollo de las competencias, los valores y las actitudes que permiten a los ciudadanos llevar vidas saludables y plenas, tomar decisiones con conocimiento de causa y responder a los desafíos locales y mundiales en base a la educación para el desarrollo sostenible y la educación para la ciudadanía mundial. En la Asamblea General de las Naciones Unidas sobre la Agenda 2030 para el Desarrollo Sostenible se refrenda el compromiso a través del Objetivo 4 «Educación de Calidad», que busca lograr una educación inclusiva, equitativa y de calidad promoviendo oportunidades de aprendizaje durante toda la vida para todos, sobre la base de que la educación es uno de los motores más poderosos y probados para garantizar el desarrollo sostenible (UN, s.f.).

Por su parte, motivado por los esfuerzos internacionales, en México, desde 1995, la educación para la sustentabilidad ya era una meta definida y estructurada al crearse el Centro de Educación y Capacitación para el Desarrollo Sustentable (CECADESU) dentro de la Secretaría de Medio Ambiente, Recursos Naturales y Pesca. El Centro perseguía el objetivo de fortalecer los contenidos ambientales de los planes y programas de estudio y de los materiales de enseñanza para los diversos niveles y modalidades de la educación, para lo cual promovió la elaboración de Planes Estatales de Educación, Capacitación y Comunicación Ambientales, en coordinación con los principales actores de la educación para la sustentabilidad en los Estados, mismos que representan un primer acuerdo estatal, con la promoción de la Federación para el establecimiento de las prioridades en el campo de la educación para el desarrollo sustentable (CECADESU, 2012).

En marzo de 2005 se firmó por parte de la Secretaría de Educación Pública y la Secretaría de Medio Ambiente y Recursos Naturales el Compromiso Nacional por la Década de la Educación para el Desarrollo Sustentable, consolidándose en 2007, al incorporar la educación para la sustentabilidad en el Plan Nacional de Desarrollo y en los Programas Sectoriales de Medio Ambiente y Recursos Naturales y Educación. Estos documentos tenían la finalidad de integrar el desarrollo sustentable en el sistema educativo nacional en todos sus niveles y modalidades (CECADESU, 2012). En el ámbito de la gestión pública, en 2005 la educación para el desarrollo sustentable también presentó avances relevantes al integrarse en los 32 planes estatales de educación, capacitación y comunicación ambiental (SEMARNAT, 2006).

Empero, en México, el esfuerzo mencionado no se ha traducido en un cambio cultural, ya que aún continúan las carencias sociales y el deterioro ambiental como consecuencia de rezagos en la legislación y la ausencia de una plataforma para la construcción de políticas públicas que resulte del análisis ciudadano desde el enfoque del desarrollo sustentable (Reyes y Bravo, 2008).

Más aún, en el actual Programa Sectorial de Educación 2013-2018, aunque se reconoce la importancia de la educación para el desarrollo sustentable, no se establecen estrategias concretas en los diferentes niveles educativos para desarrollar y fortalecer la sustentabilidad, por lo que es necesario y urgente definir un plan de acción para promover el cambio (SEP, 2013). 
Ahora bien, con la intención de coadyuvar con el reto de la educación para la sustentabilidad en México, el propósito de esta investigación es indagar en detalle el alcance de la educación para la sustentabilidad en la formación profesional de los egresados de licenciatura en las disciplinas del área de negocios. Se realizará un análisis de la percepción sobre el tema de la sustentabilidad que manifiestan tener estudiantes y profesores, lo que permitirá identificar las áreas de oportunidad para el desarrollo de una estrategia de educación transversal para la sustentabilidad aplicada en escuelas de negocios de universidades públicas y privadas en México.

Como ya se mencionó, en los Planes Nacionales de Desarrollo y programas sectoriales en México se ha reconocido a la educación como un instrumento indispensable para la formación de una cultura ambiental y para la sustentabilidad donde descansan, en buena medida, las posibilidades de un desarrollo sano y ecológicamente equilibrado. Aunque la Secretaría de Educación Pública ha realizado acciones en el ámbito de la educación básica, tiene presencia limitada en los niveles medios y superiores de la educación, razón por la cual el esfuerzo de promover la educación para la sustentabilidad desde las escuelas de negocios convierte a este proyecto de investigación interinstitucional en una actividad de colaboración de gran valor para la sociedad.

Idealmente, como lo establece la UNESCO, s.f.,b, los programas de educación profesional deben constituir, como parte de sus compromisos con la sociedad, las formas de desarrollar en los futuros profesionistas la capacidad de reflexionar y entender las consecuencias del modelo de desarrollo actual así como de proponer modelos alternativos replanteando las bases del modelo económico y social desde el enfoque de la sustentabilidad, de modo que los egresados se conviertan en detonadores de acciones concretas con un efecto multiplicador a nivel regional y global (Novo, 2009). Además, más allá de la profesión, como Rillo (2015) lo menciona, es fundamental relacionar las competencias del egresado con los principios del desarrollo sostenible, al reconocer la interconexión de los diferentes ámbitos de la vida social económica y del medio ambiente y al diseñar e implementar actividades económicas acordes con la sustentabilidad. La sustentabilidad es mucho más que el cuidado y manejo de los recursos naturales, se origina en la atención a la problemática social y su interacción con los entornos ambiental y económico propios de cada comunidad, por lo que no debe acotarse al ámbito ambiental (Quiroz y Sierra, 2008). Desde esa perspectiva, el ser humano transforma y rediseña los espacios naturales manteniendo un beneficio directo y permanente que ayuda al desarrollo de las localidades que habita.

Dado lo anterior, y en virtud de la relevancia que las áreas de los negocios tienen en la detección de oportunidades de desarrollo económico y social, consideramos que es oportuno cerciorarse en qué medida existe una estrategia educativa, definida por las escuelas de negocios, que permita a los estudiantes ser conscientes de la forma en que la actividad económica puede y debe contribuir al desarrollo sustentable.

Para ello se plantearon las siguientes preguntas de investigación: ¿Qué evidencia existe de la presencia de propósitos a favor de la sustentabilidad en documentos institucionales y planes de estudio de las carreras del área de los negocios? ¿En qué grado los profesores y estudiantes de estas disciplinas son conscientes de la importancia y trascendencia de la sustentabilidad en el área de los negocios?

Para responder las preguntas anteriores se invitó a participar en este estudio a las escuelas de negocios de universidades públicas y privadas en México que forman parte de la comunidad virtual CUDI (ver sección de Metodología). Inicialmente se realizó un análisis de los contenidos de documentos institucionales y de planes de estudios de dos carreras en el área de los negocios (licenciado en administración de empresas [LAE] y licenciado en contaduría pública [LCP] o sus equivalentes) para identificar la presencia de propuestas a favor de la sustentabilidad; posteriormente se llevó a cabo una consulta a estudiantes y profesores de profesional con la finalidad de 
conocer su percepción en este tema. A partir de los resultados emanados del análisis se identificaron las carencias existentes y finalmente, a manera de conclusión, se identifican los elementos que podrían ser la base para el diseño de propuestas para la capacitación de profesores y estudiantes de escuelas de negocios.

\section{Marco teórico}

Las múltiples evidencias de los efectos dañinos del modelo de desarrollo económico vigente enfocado en un crecimiento supuestamente ilimitado, como son el cambio climático, la pérdida de biodiversidad, la contaminación, etc., demuestran que la sustentabilidad es un problema de carácter fundamentalmente económico y social con efectos en el medio ambiente natural, que a su vez afectan la vida económica y social. Por tal motivo, la educación debe evolucionar de modo que habilite a los educandos para orientar sus acciones hacia los objetivos que demanda una forma de vida sustentable. La propuesta educativa para la sustentabilidad deberá desarrollar en el educando la dimensión de la conciencia ambiental que le permita ir más allá de la mera conservación, considerando al medio ambiente como un elemento intrínseco para nuestra supervivencia (Meira, 2006; Morín, 1999). Para implementar las ideas de la sustentabilidad en los modelos de negocios será necesario propiciar, a través de la innovación, un giro en los modelos de negocios en los que se privilegien los productos duraderos y el trabajo decente ${ }^{1}$, persiguiendo la equidad social a través de la redistribución de la riqueza y el desarrollo regional.

En las escuelas de negocios la innovación educativa está integrada por los cambios y mejoras en la docencia y en la investigación, por la actualización docente y profesional, y por la adecuación y adaptación de los contenidos de las diversas disciplinas al entorno ambiental y las necesidades que demanda la sociedad (Fisher, Sax y Grove, 2000; Farías Martínez y Lavín-Verástegui, 2012).

Cabe señalar que además del enfoque hacia los conocimientos existentes y por generar, la sustentabilidad presupone un enfoque ético hacia la actividad económica a nivel societal y de empresa. Actuar de forma sustentable significa modificar las conductas propias en pro de otras personas, mientras que las teorías más en boga en las escuelas de negocios se basan en el beneficio propio amoral (véase Donaldson, 2002). Aunque es cuestionable si la educación brindada a jóvenes adultos aún puede impactar en su disposición ética fundamental, un programa educativo que enfatice la sustentabilidad debería al menos ayudar a corregir los puntos ciegos que llevan a una eticidad restringida (Bazerman y Tenbrunsel, 2011), es decir, la incapacidad de percatarse de las consecuencias negativas que las propias acciones pueden tener en otras personas. Idealmente, el ambiente universitario puede despertar en los jóvenes una pasión por la sustentabilidad (Shrivastava, 2010), es decir, ayudaría a vincular lo emocional con lo racionalmente deseable.

Las escuelas de negocios en el mundo han iniciado un proceso de evolución en los planes de estudios de modo que proporcionen las herramientas para que sus egresados sean capaces de llevar a cabo una gestión basada en una reflexión ética sobre el actual modelo económico de desarrollo (Garner y Stead, 2010). El llamado urgente a modificar la educación para los negocios, en función de los principios y valores ambientales y ciudadanos a través de un aprendizaje vivencial, detona la innovación de las metodologías de aprendizaje que permitirán el cambio para redefinir la educación empresarial (Alcaraz y Thiruvattal, 2010; Selwyn, 2004, citado por Gros y Contreras, 2006).

\footnotetext{
1 «El trabajo decente promovido por la OIT implica generar suficientes puestos de trabajo teniendo como requisito indispensable que sean empleos productivos y de calidad, y que las trabajadoras y los trabajadores los ocupen en condiciones de libertad, igualdad, seguridad y dignidad humana» (OIT, 2016, p. 1).
} 
La Organización de las Naciones Unidas convoca a la comunidad empresarial a adoptar un estilo de gestión basado en los valores que fueron definidos en el Pacto Mundial relativos a los derechos humanos, a las relaciones laborales, a la lucha contra la corrupción y a la protección del medio ambiente (UNGC, 2009). Adicionalmente, invita a las empresas a patrocinar la llamada educación global, educación para todos y para todas. Aunque desde la conceptualización más general el Pacto Mundial oriente a las empresas miembros a buscar la equidad, el trabajo decente y la protección ambiental, es importante tener cautela respecto a la perspectiva del beneficio de la educación global. Ban Ki-moon menciona que la expectativa de la educación global es reproducir ciudadanos calificados, y por ende con mayores ingresos, que demandarán nuevos productos y servicios, consolidando al actual modelo de mercado, sin condescender con el principio de mesura en el consumo que propone la educación para la sustentabilidad (UNGC, 2013).

La propuesta de modificar la educación para los negocios en función de la evolución de las ideas acerca de la ciudadanía corporativa, la responsabilidad social corporativa y la sostenibilidad tiene el objetivo de proporcionar el marco conceptual de una gestión empresarial que permita el cambio del paradigma del máximo beneficio económico individual hacia el del beneficio de la sociedad en su conjunto (Alonso-Almeida, Rodríguez, Cortez y Abreu, 2012; Alcaraz y Thiruvattal, 2010).

Para lograr lo anterior, se requieren actividades de enseñanza-aprendizaje que promuevan una acción socialmente responsable que parta de un conocimiento académicamente riguroso, apoyada en la reflexión crítica y puesta en práctica mediante enfoques innovadores. Asimismo, como parte de la promoción de la ciudadanía a través de la educación transversal, se busca ayudar al estudiante a descubrir e interiorizar el valor social de su vocación profesional (Sandrea y Reyes, 2010), fortaleciendo su identidad como profesionista.

Entre las propuestas que han surgido para facilitar la innovación educativa (ANFECA, 2007) se encuentran la creación y la puesta en marcha de programas de capacitación docente y la creación y el diseño de prácticas educativas tales como el aprendizaje a través de estudios de caso que presenten dilemas de sustentabilidad (Haugh y Talwar, 2010), el aprendizaje basado en el servicio (Amat y Moliner, 2010), el uso adecuado de las tecnologías de información, así como el manejo y análisis de datos mediante simuladores (Heuer, 2010), además de la creación de espacios de interacción y vinculación que favorezcan la transversalidad, como por ejemplo a través de escuelas prácticas en temas relacionados con la sustentabilidad (Benn y Martin, 2010).

A partir de lo anterior, los autores de esta investigación consideramos necesario llevar a cabo un diagnóstico de la comprensión y el entendimiento que profesores y estudiantes en las áreas de negocios en México tienen respecto al concepto de la sustentabilidad, de modo que sea la base de la formulación de la propuesta educativa con miras a la sustentabilidad. En la siguiente sección se detalla la metodología utilizada para conocer el estado actual de un grupo de universidades en México.

\section{Metodología}

La metodología que se utilizó en esta investigación fue diseñada para hacer una indagatoria sobre: 1) la presencia de propósitos en torno a la sustentabilidad, manifiestos a través de menciones en documentos institucionales y en los planes de estudios de las carreras profesionales en el área de los negocios, así como también 2) la percepción que tienen estudiantes y profesores de las universidades participantes en temas de sustentabilidad. Para ello se llevó a cabo un estudio empírico mediante la identificación de contenidos relativos a la sustentabilidad y el análisis de la percepción sobre el concepto de sustentabilidad relacionado con los negocios. 
Para la revisión de los textos de visión y misión se empleó el método de análisis de contenidos, que permite identificar los componentes semánticos en todo tipo de mensajes. El método permite interpretarlos sistemáticamente a fin de establecer inferencias con validez objetiva (López Noguero, 2002; Wu, Huang, Kuo y Wu, 2010). Por otra parte, para el análisis de las percepciones de estudiantes y profesores se emplearon tablas de frecuencias relativas, que permiten analizar el comportamiento de un fenómeno determinado dentro de un contexto particular (Kerlinger y Lee, 2002). Con el propósito de hacer generalizaciones sobre la población de estudio se llevaron a cabo pruebas inferenciales de comparación entre proporciones utilizando la prueba normal estándar.

\section{Conformación del grupo de universidades participantes}

El 27 de septiembre de 2013 se lanzó una invitación abierta para participar en este proyecto de investigación a universidades que pertenecen a la Comunidad de Contaduría y Negocios a través de la Red Nacional de Educación e Investigación (RNEI) perteneciente a la Corporación Universitaria para el Desarrollo de Internet (CUDI). Esta red de colaboración universitaria está conformada por 18 universidades mexicanas entre públicas y privadas e identidades de educación superior $^{2}$. Las instituciones de educación superior que decidieron libremente colaborar con el proyecto son las siguientes: Instituto Tecnológico de Tijuana; Tecnológico de Monterrey, Campus Monterrey; Universidad de Guadalajara/CU Lagos; Universidad Autónoma de Tamaulipas; Universidad del Valle de Atemajac; Universidad Iberoamericana Puebla; Universidad Juárez del Estado de Durango; Universidad La Salle; Universidad Politécnica de Victoria, y Universidad Veracruzana.

Es importante señalar que aunque las universidades participantes no fueron seleccionadas aleatoriamente, la muestra se conformó por instituciones tanto públicas como privadas, ubicadas en las zonas Norte y Centro del país.

Con la intención de responder a las dos preguntas formuladas en esta investigación se planteó la realización de las siguientes etapas del análisis.

\section{Primera etapa. Revisión de las menciones de sustentabilidad en documentos oficiales}

1. Se extrajeron, a partir de los sitios web de cada universidad ${ }^{3}$, los textos concernientes a la visión y la misión institucionales así como los planes de estudio de las carreras licenciado en administración de empresas (LAE) y licenciado en contaduría pública (LCP) o equivalentes.

2. Se definieron las categorías bajo las cuales se detectaría la presencia de los conceptos de sustentabilidad en los documentos evaluados, siendo estos los siguientes:

\footnotetext{
2 También pertenecen a la red CUDI 28 Centros Conacyt y 92 Institutos Tecnológicos y Universidades Politécnicas.

3 Direcciones de las páginas web:

Instituto Tecnológico de Tijuana <http://tectijuana.edu.mx/>

Tecnológico de Monterrey, Campus Monterrey <http://www.itesm.mx/>

Universidad de Guadalajara/CU Lagos <http://www.udg.mx/es/secciones/culagos>

Universidad Autónoma de Tamaulipas <http://www.uat.edu.mx/>

Universidad del Valle de Atemajac <http://www.univa.mx/>

Universidad Iberoamericana Puebla <http://www.iberopuebla.mx/>

Universidad Juárez del Estado de Durango <http://www.ujed.mx/>

Universidad La Salle <http://www.lasalle.mx/>

Universidad Politécnica de Victoria <http://www.upvictoria.edu.mx/>

Universidad Veracruzana <http://www.uv.mx/>
} 
- Eje ambiental: en los casos en los que se mencione la conservación y la protección del medio ambiente.

- Eje social: cuando se hable de ética, responsabilidad social, equidad, inclusión, etc.

- Eje económico: cuando se expresen compromisos hacia la redistribución de la riqueza, comercio justo, control del consumo, etc.

- Los conceptos «desarrollo sostenible» o «desarrollo sustentable», que se toman como sinónimos, se consideraron como afirmaciones no diferenciadas.

3. La lectura de los textos de cada universidad la realizaron dos codificadores. Los codificadores fueron estudiantes de maestría en las áreas de negocios. A ambos se les enviaron por correo electrónico los documentos a evaluar, los criterios para hacer la identificación y la tabla para recopilar los resultados. Dado que los codificadores trabajaron de manera aislada, se considera que existe independencia entre sus respuestas. Las tablas de resultados fueron enviadas a la primera autora vía correo electrónico para su compilación y análisis.

A partir de las tablas de reconocimiento de contenidos se identificaron las coincidencias y discrepancias entre las respuestas de los codificadores. Para validar estadísticamente el análisis, se calculó el coeficiente de acuerdo con el alfa de Krippendorff (Krippendorff, 1997), siendo $\alpha=0.8297$, por lo que el acuerdo obtenido es aproximadamente un $83 \%$ superior a un acuerdo ocurrido gracias al azar. Krippendorff (1997) menciona que una fiabilidad superior a 0.8 es aceptable, por lo tanto se consideró válida la interpretación de los resultados que se muestran en la siguiente sección. Los pocos segmentos de texto donde resultaron clasificaciones no coincidentes fueron analizados de nuevo por los autores, quienes consideraron que estaban poco claros y los asignaron a la categoría de no diferenciados.

\section{Segunda etapa. Percepción sobre el concepto de sustentabilidad en los negocios}

\section{Diseño y validación del instrumento}

Partiendo de la revisión de diversos documentos (CONAGUA, s.f.; Gordon, 1998; MurgaMenoyo, 2008; OCDE, 2008) se establecieron dos dimensiones de análisis a partir de las cuales se construyó una matriz con los siguientes elementos:

1) Ejes de la sustentabilidad:
a. Ambiental.
b. Social.
c. Económico.

2) Nivel de reconocimiento del concepto:
a. Comprende el concepto.
b. Lo percibe en el ámbito cotidiano (como ciudadano).
c. Lo percibe en el ámbito profesional (como profesionista).

Para cada intersección de la matriz conformada con las dos dimensiones anteriores se elaboraron de 2 a 3 reactivos, dando un total de 22. El nivel de acuerdo respecto a cada reactivo fue medido en escala Likert (1, «totalmente en desacuerdo», hasta 5, «muy de acuerdo»).

El instrumento se aplicó inicialmente a 40 profesores y a 75 estudiantes de las 10 universidades participantes con el fin de validarlo estadísticamente. Como resultado se obtuvo el alfa de Cronbach $\alpha=0.803$ para la encuesta de estudiantes y $\alpha=0.897$ para la encuesta de profesores. Dado que 
los valores obtenidos para ambos instrumentos superan el valor crítico de 0.7 , se considera que el instrumento tiene validez estadística para ambos grupos (Tavakol y Dennick, 2011).

\section{Muestreo}

Para el grupo de estudiantes se realizó un muestreo por conglomerados, siendo el conglomerado el salón de clase. Los salones encuestados de cada universidad fueron seleccionados al azar a partir del total de salones en los que se imparten materias a estudiantes del área de negocios. La aplicación fue supervisada en salas con computadoras debido a que se contestó en la plataforma Qualtrics que opera en línea. El tamaño de la muestra para los estudiantes se calculó, asumiendo una población infinita, con una significancia y error de estimación iguales al 10\%, obteniéndose una muestra requerida de 68 estudiantes por universidad; la mayoría de las universidades excedió este valor.

Para el grupo de profesores, el tamaño de la muestra se calculó asumiendo una población finita, con una significancia y error de estimación igual al 10\%, lo anterior debido a que el grupo de profesores es mucho menos numeroso que el grupo de estudiantes, obteniéndose un tamaño de muestra requerido igual a 18 profesores para cada universidad. Se invitó por vía electrónica a participar a todos los profesores de la facultad de cada universidad; en la mayoría de las universidades se excedió el tamaño de muestra establecido. Al igual que el grupo de los estudiantes, la encuesta se contestó en la plataforma Qualtrics.

\section{Análisis estadístico}

En un inicio, los datos obtenidos a partir de la muestra fueron condensados y ordenados. Posteriormente, los resultados de las percepciones de ambos grupos, estudiantes y profesores, fueron analizados mediante la comparación de las proporciones de respuestas a favor o en contra de cada reactivo.

Para facilitar la interpretación de la comparación entre las percepciones sobre la sustentabilidad, los valores 1 y 2 de la escala Likert fueron considerados como «en desacuerdo», el valor 3 de la escala se consideró como «indiferente» y los valores 4 y 5 de la escala fueron considerados como «de acuerdo» respecto a las aseveraciones formuladas.

Los contrastes de hipótesis para las proporciones se realizaron mediante la prueba estadística paramétrica normal estándar. La condición de normalidad de los estadísticos se cumplió gracias al teorema del límite central por tener tamaños de muestra mucho mayores a 30. El análisis estadístico se realizó utilizando el software NCSS 2007.

\section{Resultados}

\section{Primera etapa. Análisis de contenidos}

La tabla 1 muestra el porcentaje, del total de universidades evaluadas, donde se encontraron menciones de cada uno de los conceptos de sustentabilidad, bajo los criterios que fueron definidos para los tres ejes: ambiental, social y económico, en los documentos de visión, misión y planes de estudios para las carreras licenciado en administración de empresas (LAE) y licenciado en contaduría pública (LCP). Además de las menciones sin especificación que se refieren al concepto genérico de desarrollo sustentable o desarrollo sostenible.

Como se observa en la tabla 1, el eje social es el que recibe la mayor cantidad de menciones en todos los documentos. Es notable que el eje económico sea mencionado solamente por el 
Tabla 1

Frecuencias relativas en las menciones de los conceptos de sustentabilidad

\begin{tabular}{lllll}
\hline Eje de sustentabilidad & \multicolumn{2}{c}{ Documentos institucionales } & \multicolumn{2}{c}{ Plan de estudios } \\
\cline { 2 - 5 } & Visión & Misión & LAE & LCP \\
\hline Ambiental & $14 \%$ & $57 \%$ & - & - \\
Social & $57 \%$ & $86 \%$ & $86 \%$ & $71 \%$ \\
Económico & - & $29 \%$ & - & - \\
Sin especificación & $29 \%$ & $29 \%$ & $57 \%$ & $29 \%$ \\
\hline
\end{tabular}

Fuente: elaboración propia a partir de los resultados del análisis.

$29 \%$ de las universidades, y estas menciones se hagan solo en documentos institucionales. Con relación al eje ambiental, es mencionado hasta por el $57 \%$ de las universidades, pero solamente en documentos institucionales, pues no se identificó ninguna mención en los planes académicos.

Finalmente, existe una amplia proporción, casi la tercera parte de las universidades, que mencionaron la sustentabilidad como un aspecto general no diferenciado. Lo anterior muestra que existe cierta incoherencia que, aunque propia de la ambigüedad del concepto, provoca que no logren puntualizarse las menciones dirigidas a la sustentabilidad en los documentos institucionales ni en los planes de estudios de las carreras que fueron analizadas en esta investigación.

En la tabla 2 se muestran las frases que se relacionan con los conceptos de sustentabilidad expresadas en la visión, misión y planes de estudios.

Como puede observarse, el eje social es en el que se identificaron la mayor cantidad de conceptos. Este resultado da evidencia de que las presentaciones institucionales de las universidades, inspiradas en los ideales de sus directivos, reflejan la concepción de los problemas de corte social, por lo que manifiestan explícitamente compromisos en pro de sumarse al esfuerzo por contribuir a la sustentabilidad desde el ámbito social. También se puede observar que no se visualiza un involucramiento directo con los ejes ambiental y económico de la sustentabilidad, sino que los presentan de manera no diferenciada. En la sección de conclusiones se hacen algunas reflexiones respecto a los resultados anteriores.

\section{Segunda etapa. Percepción sobre el concepto de sustentabilidad}

Inicialmente se llevó a cabo un análisis demográfico de los participantes. La tabla 3 muestra las características de los grupos conformados por 863 estudiantes y 192 profesores, ambos pertenecientes a las 10 universidades participantes.

La tabla 3 muestra que en del grupo de estudiantes hay una mayor participación de mujeres, casi un $20 \%$ superior a la proporción de hombres. Por su parte, la comparación entre universidades públicas y privadas presentó proporciones prácticamente iguales. Con relación al grupo de profesores, hubo una mayor proporción de participantes hombres, aproximadamente un 14\% superior a la proporción de mujeres, la mayoría de los participantes con grado académico de maestría y dedicados fundamentalmente a actividades académicas. Los datos anteriores podrían ser motivo de hacer comparativos entre las percepciones diferenciadas por género, tipo de universidad, grado académico y principal actividad profesional. En la sección de conclusiones se harán recomendaciones para considerar dichas variables en futuros análisis sobre este tema, ya que en esta investigación no se hicieron comparativos con base en dichos criterios. 
Tabla 2

Textos de las menciones del concepto de sustentabilidad

\begin{tabular}{|c|c|c|c|c|}
\hline \multirow{2}{*}{$\begin{array}{l}\text { Eje de la } \\
\text { sustentabilidad }\end{array}$} & \multicolumn{2}{|c|}{ Documentos institucionales } & \multicolumn{2}{|c|}{ Planes de estudios } \\
\hline & Visión & Misión & LAE & LCP \\
\hline Ambiental & $\begin{array}{l}\text { Conciencia } \\
\text { ecológica }\end{array}$ & $\begin{array}{l}\text { Sustentabilidad } \\
\text { ambiental. Uso sostenible } \\
\text { de los recursos naturales }\end{array}$ & & \\
\hline Social & $\begin{array}{l}\text { Desarrollo social } \\
\text { de la región. } \\
\text { Promover la } \\
\text { equidad. Busca el } \\
\text { bien común. } \\
\text { Compromiso del } \\
\text { capital humano }\end{array}$ & $\begin{array}{l}\text { Justicia social. Formación } \\
\text { humanística. } \\
\text { Responsabilidad y } \\
\text { compromiso social. } \\
\text { Desarrollo social. Calidad } \\
\text { humana. Solución a } \\
\text { problemas de impacto } \\
\text { social. Bienestar de la } \\
\text { población. Compromiso } \\
\text { con la transformación de } \\
\text { la comunidad }\end{array}$ & $\begin{array}{l}\text { Sensible a } \\
\text { necesidades sociales. } \\
\text { Actitud ética. } \\
\text { Responsabilidad } \\
\text { social. Ambiente } \\
\text { laboral de equidad y } \\
\text { respeto. Fomentar el } \\
\text { bienestar social }\end{array}$ & $\begin{array}{l}\text { Bienestar social. } \\
\text { Compromiso y } \\
\text { responsabilidad } \\
\text { social. Actitud } \\
\text { ética. Desarrollar } \\
\text { proyectos que } \\
\text { resuelvan } \\
\text { problemas bajo } \\
\text { principios de } \\
\text { responsabilidad } \\
\text { social }\end{array}$ \\
\hline Económico & & $\begin{array}{l}\text { Convivencia democrática. } \\
\text { Propiedad colectiva. } \\
\text { Agentes de cambio en el } \\
\text { entorno local y global }\end{array}$ & & \\
\hline Sin especificación & $\begin{array}{l}\text { Desarrollo } \\
\text { sustentable }\end{array}$ & Desarrollo sustentable & $\begin{array}{l}\text { Diseñar y gestionar } \\
\text { proyectos } \\
\text { sustentables. } \\
\text { Emprendimiento } \\
\text { sustentable }\end{array}$ & $\begin{array}{l}\text { Fomentar el } \\
\text { desarrollo } \\
\text { sustentable }\end{array}$ \\
\hline
\end{tabular}

Fuente: elaboración propia a partir de los resultados del análisis.

Tabla 3

Características demográficas de los encuestados

\begin{tabular}{ll}
\hline Estudiantes & \\
Género & \\
Hombres & $41 \%$ \\
Mujeres & $59 \%$ \\
Universidad & \\
Públicas & $51 \%$ \\
Privadas & $49 \%$ \\
Profesores & \\
Género & \\
Hombres & \\
Mujeres & $57 \%$ \\
Grado & $43 \%$ \\
Maestría & \\
Doctorado & $60 \%$ \\
Otro & $21 \%$ \\
Principal actividad & $19 \%$ \\
Docencia & \\
Otras & $79 \%$ \\
\end{tabular}

Fuente: elaboración propia. 
Tabla 4

Análisis comparativo entre profesores y estudiantes respecto a la comprensión del concepto de sustentabilidad

\begin{tabular}{llll}
\hline & Profesores & Estudiantes & Sig. \\
\hline Eje ambiental & & & \\
Para entender los problemas de la sustentabilidad se necesita cursar & materias relacionadas con el medio \\
ambiente como la ecología y la biología & & - \\
Desacuerdo & 0.28 & 0.34 & - \\
Indiferente & 0.23 & 0.25 & 0.2057 \\
De acuerdo & 0.49 & 0.41 &
\end{tabular}

La sustentabilidad se relaciona solamente con el deterioro del medio ambiente como por ejemplo el cambio climático
Desacuerdo
0.11
0.54
Indiferente
0.16
0.26
De acuerdo
0.73
0.20
$-$
0.000

Los problemas del medio ambiente pueden resolverse con innovación y tecnología

$\begin{array}{lll}\text { Desacuerdo } & 0.12 & 0.12 \\ \text { Indiferente } & 0.27 & 0.21 \\ \text { De acuerdo } & 0.61 & 0.67\end{array}$

0.12

0.21

0.4693

Eje social

La sustentabilidad se relaciona con la falta de equidad

$\begin{array}{llll}\text { Desacuerdo } & 0.36 & 0.33 & - \\ \text { Indiferente } & 0.23 & 0.39 & - \\ \text { De acuerdo } & 0.41 & 0.28 & 0.0145\end{array}$

Si se disminuyera la pobreza sería más fácil conseguir la sustentabilidad

$\begin{array}{llll}\text { Desacuerdo } & 0.22 & 0.27 & - \\ \text { Indiferente } & 0.23 & 0.29 & - \\ \text { De acuerdo } & 0.54 & 0.44 & 0.1264\end{array}$

Los problemas de la sustentabilidad afectan más a los países pobres

$\begin{array}{lll}\text { Desacuerdo } & 0.19 & 0.23 \\ \text { Indiferente } & 0.16 & 0.28 \\ \text { De acuerdo } & 0.64 & 0.50\end{array}$

0.50

$-$

0.0556

Eje económico

De los tres ejes de la sustentabilidad (económico, social y ambiental), el que debe ser atendido con mayor urgencia es el económico
Desacuerdo
0.39
0.38
0.8498
Indiferente
0.29
0.34
De acuerdo
0.32
0.27
La sustentabilidad se relaciona con el estilo de consumo de la sociedad

$\begin{array}{llll}\text { Desacuerdo } & 0.07 & 0.08 & - \\ \text { Indiferente } & 0.10 & 0.20 & - \\ \text { De acuerdo } & 0.83 & 0.72 & 0.2407\end{array}$

Para entender los problemas de la sustentabilidad se necesita conocer los antecedentes del desarrollo económico
Desacuerdo
0.15
0.18
Indiferente
0.22
De acuerdo
0.63
0.34
0.48
0.0372

La significancia (Sig.) para la comparación de proporciones a partir de la prueba normal estándar representa la probabilidad de que las proporciones comparadas sean iguales. Se rechaza la igualdad para probabilidades menores al $10 \%$.

Fuente: elaboración propia.

\section{a) Respecto a la comprensión del concepto de sustentabilidad}

La tabla 4 muestra los resultados de las proporciones de profesores y estudiantes que manifestaron estar de acuerdo respecto a cada uno de los reactivos correspondientes a cada eje de la sustentabilidad, para la categoría Comprende el concepto de sustentabilidad. Se hace una 
diferenciación entre profesores y estudiantes para todas las categorías a fin de detectar la existencia de diferencias significativas entre ambos grupos. Al respecto cabe presentar dos hipótesis explicativas: la primera es que las diferencias podrían ser inducidas por las acciones que la Secretaría de Educación Pública ha llevado a cabo en el tema de la sustentabilidad en el nivel de educación básica; la segunda hipótesis se basaría en el nivel de educación general, que por definición es mayor en profesores que en estudiantes; esta hipótesis corresponde a las percepciones de la mayoría en ambos grupos de encuestados.

Cabe señalar que la comparación de proporciones para cada aseveración entre profesores y estudiantes se llevó a cabo solo para el nivel (en desacuerdo, indiferente, de acuerdo) con la mayor frecuencia. Para el resto de los niveles no hubo una estimación de la significancia, por lo que su correspondiente espacio en la tabla fue ocupado por un $(-)$.

Partiendo los resultados de la tabla 4, se puede observar:

Respecto a los profesores. El $73 \%$ percibe que la sustentabilidad es un problema solamente del medio ambiente. Se piensa que la ciencia y la tecnología tienen mayor participación en la solución de los problemas de sustentabilidad, aunque falta conocer más sobre el medio ambiente. Una proporción del $41 \%$ percibe una relación entre la equidad social y la sustentabilidad. Aproximadamente la mitad considera que la disminución de la pobreza aumentaría la posibilidad de conseguir la sustentabilidad, mientras que el $64 \%$ considera que los problemas de la sustentabilidad afectan más a los países pobres. Solamente el $39 \%$ percibe claramente la urgencia de atender al eje económico de la sustentabilidad, aunque el $83 \%$ percibe la relación adversa entre el consumo y la sustentabilidad. El $63 \%$ percibe la necesidad de conocer el impacto de la economía en la sustentabilidad.

Respecto a los estudiantes. El 54\% no percibe que la sustentabilidad sea exclusiva del medio ambiente. Se tienen percepciones muy cercanas a las de los profesores respecto a la ciencia y la tecnología, también perciben la necesidad de conocer más sobre el medio ambiente. El 39\% no tiene una opinión decidida sobre la relación entre equidad y sustentabilidad. No existe una diferencia significativa respecto a los profesores en cuanto a la percepción de la pobreza como obstáculo para alcanzar la sustentabilidad. Una parte comparativamente menor del estudiantado (50\%) piensa que los problemas de la sustentabilidad afectan más a los pobres. Respecto al eje económico, solo difieren significativamente del grupo de profesores en que perciben en menor proporción (48\%) la necesidad de conocer el impacto de la economía en la sustentabilidad.

\section{b) Respecto a la percepción del concepto de la sustentabilidad en el ámbito cotidiano}

Los resultados de la comparación entre las proporciones que indican estar de acuerdo con cada uno de los reactivos correspondientes a cada eje de la sustentabilidad para la categoría Percepción sobre el involucramiento como ciudadano con la sustentabilidad se muestran en la tabla 5. Al igual que la tabla 4, la comparación de proporciones para cada aseveración entre profesores y estudiantes se llevó a cabo solo para el nivel (en desacuerdo, indiferente, de acuerdo) con la mayor frecuencia. Para el resto de los niveles no hubo una estimación de la significancia, por lo que su correspondiente espacio en la tabla fue ocupado por un $(-)$.

Partiendo del análisis comparativo de proporciones que se muestran en la tabla 5 se observa:

Respecto a los profesores. Una mayoría contundente manifiesta cuidar los recursos como el agua $(91 \%)$ y la energía (86\%), lo que en el segundo caso representa una proporción significativamente mayor que los estudiantes. Una mayoría abrumadora (89\%) reconoce que actuar con principios éticos y morales contribuye a la sustentabilidad. El $64 \%$ reconoce a la educación como un motor para la sustentabilidad. En proporciones casi iguales consideran más importante reciclar 
Tabla 5

Análisis comparativo entre proporciones profesores/estudiantes para la actitud hacia la sustentabilidad como ciudadano

\begin{tabular}{|c|c|c|c|}
\hline & Profesores & Estudiantes & Sig. \\
\hline \multicolumn{4}{|l|}{ Eje ambiental } \\
\hline \multicolumn{4}{|c|}{ Entre mis hábitos cotidianos está cuidar el agua } \\
\hline Desacuerdo & 0.04 & 0.05 & - \\
\hline Indiferente & 0.05 & 0.17 & - \\
\hline De acuerdo & 0.91 & 0.77 & 0.1466 \\
\hline \multicolumn{4}{|c|}{ Doy preferencia a la compra de electrodomésticos que consumen menos energía } \\
\hline Desacuerdo & 0.07 & 0.12 & - \\
\hline Indiferente & 0.06 & 0.20 & - \\
\hline De acuerdo & 0.86 & 0.68 & 0.048 \\
\hline \multicolumn{4}{|l|}{ Eje social } \\
\hline \multicolumn{4}{|c|}{ Yo contribuyo a resolver los problemas derivados de la sustentabilidad (por ejemplo: pobreza, deterioro del } \\
\hline \multicolumn{4}{|c|}{ medio ambiente, corrupción) cuando actúo bajo principios éticos y morales } \\
\hline Desacuerdo & 0.04 & 0.11 & - \\
\hline Indiferente & 0.08 & 0.22 & - \\
\hline De acuerdo & 0.89 & 0.67 & 0.0158 \\
\hline \multicolumn{4}{|c|}{ Una persona con mayor nivel educativo será más capaz de tomar decisiones en favor de la sustentabilidad } \\
\hline Desacuerdo & 0.18 & 0.17 & - \\
\hline Indiferente & 0.19 & 0.21 & - \\
\hline De acuerdo & 0.64 & 0.63 & 0.8922 \\
\hline \multicolumn{4}{|l|}{ Eje económico } \\
\hline \multicolumn{4}{|c|}{ Considero que es más importante reciclar materiales que disminuir el consumo de los productos que generan } \\
\hline \multicolumn{4}{|l|}{ desperdicio } \\
\hline Desacuerdo & 0.34 & 0.28 & - \\
\hline Indiferente & 0.28 & 0.25 & - \\
\hline De acuerdo & 0.39 & 0.48 & 0.164 \\
\hline \multicolumn{4}{|c|}{ Los problemas de la sustentabilidad solo pueden resolverse con esfuerzos globales } \\
\hline Desacuerdo & 0.13 & 0.15 & - \\
\hline Indiferente & 0.12 & 0.23 & - \\
\hline De acuerdo & 0.75 & 0.61 & 0.0934 \\
\hline
\end{tabular}

La significancia (Sig.) para la comparación de proporciones a partir de la prueba normal estándar representa la probabilidad de que las proporciones comparadas sean iguales. Se rechaza la igualdad para probabilidades menores al $10 \%$.

Fuente: elaboración propia.

materiales (39\%) que disminuir la generación de materiales de desecho (34\%). El 75\% considera que los problemas de sustentabilidad deben resolverse de manera global.

Respecto a los estudiantes. La gran mayoría manifiesta cuidar los recursos como el agua (77\%) y la energía (68\%). Dos terceras partes del estudiantado reconocen la responsabilidad moral para con la sustentabilidad, significativamente menos que los profesores, mientras que no difieren casi en su apreciación por el rol de la educación en la sustentabilidad. El 48\% considera más importante el reciclado de materiales que la diminución en el consumo. En menor proporción que los profesores $(61 \%)$, consideran que se requieren esfuerzos globales para resolver los problemas de sustentabilidad.

\section{c) Respecto a la percepción en el ámbito profesional}

Finalmente, los resultados de la comparación entre las proporciones a favor, respecto a cada uno de los reactivos correspondientes a los ejes de la sustentabilidad para la categoría Percepción sobre la relación de la sustentabilidad con el ámbito profesional, se muestran en la tabla 6. Al 
Tabla 6

Análisis comparativo entre proporciones profesores/estudiantes respecto a la actitud como profesionista hacia la sustentabilidad

\begin{tabular}{|c|c|c|c|}
\hline & Profesores & Estudiantes & Sig. \\
\hline \multicolumn{4}{|l|}{ Eje ambiental } \\
\hline \multicolumn{4}{|c|}{ La naturaleza debe conservarse porque provee la materia prima para las industrias } \\
\hline Desacuerdo & 0.32 & 0.18 & - \\
\hline Indiferente & 0.16 & 0.19 & - \\
\hline De acuerdo & 0.52 & 0.63 & 0.1593 \\
\hline \multicolumn{4}{|c|}{ La sustentabilidad de la empresa depende de la sustentabilidad del medio ambiente } \\
\hline Desacuerdo & 0.17 & 0.20 & \\
\hline Indiferente & 0.24 & 0.34 & \\
\hline De acuerdo & 0.59 & 0.46 & 0.0627 \\
\hline \multicolumn{4}{|l|}{ Eje social } \\
\hline \multicolumn{4}{|c|}{$\begin{array}{l}\text { Las prácticas laborales que promueven la igualdad de oportunidades ayudan a que la empresa permanezca a } \\
\text { través del tiempo }\end{array}$} \\
\hline Desacuerdo & 0.10 & 0.08 & - \\
\hline Indiferente & 0.15 & 0.24 & - \\
\hline De acuerdo & 0.75 & 0.68 & 0.4276 \\
\hline \multicolumn{4}{|c|}{ La existencia de un código de conducta dentro de las organizaciones favorece su sustentabilidad } \\
\hline Desacuerdo & 0.06 & 0.08 & - \\
\hline Indiferente & 0.16 & 0.22 & - \\
\hline De acuerdo & 0.77 & 0.70 & 0.4293 \\
\hline \multicolumn{4}{|c|}{ La corrupción disminuye las oportunidades para que las empresas puedan ser sustentables } \\
\hline Desacuerdo & 0.13 & 0.18 & - \\
\hline Indiferente & 0.10 & 0.24 & - \\
\hline De acuerdo & 0.76 & 0.58 & 0.0277 \\
\hline \multicolumn{4}{|l|}{ Eje económico } \\
\hline \multicolumn{4}{|c|}{ Una justa distribución de los beneficios de la empresa a los accionistas favorece la sustentabilidad } \\
\hline Desacuerdo & 0.30 & 0.16 & - \\
\hline Indiferente & 0.27 & 0.31 & - \\
\hline De acuerdo & 0.43 & 0.53 & 0.1548 \\
\hline \multicolumn{4}{|c|}{ Los problemas relacionados con la sustentabilidad solo se podrán resolver si buscamos alternativas } \\
\hline \multicolumn{4}{|c|}{ diferentes de hacer negocios } \\
\hline Desacuerdo & 0.20 & 0.28 & - \\
\hline Indiferente & 0.18 & 0.29 & - \\
\hline De acuerdo & 0.62 & 0.43 & 0.0057 \\
\hline
\end{tabular}

La significancia (Sig.) para la comparación de proporciones a partir de la prueba normal estándar representa la probabilidad de que las proporciones comparadas sean iguales. Se rechaza la igualdad para probabilidades menores al $10 \%$.

Fuente: elaboración propia.

igual que en los casos anteriores, la comparación de proporciones para cada aseveración entre profesores y estudiantes se llevó a cabo solo para el nivel (en desacuerdo, indiferente, de acuerdo) con la mayor frecuencia. Para el resto de los niveles no hubo una estimación de la significancia por lo que el espacio en la tabla fue ocupado por un (-).

Partiendo de la tabla 6 se puede observar que:

Respecto a los profesores. Más de la mitad perciben que la naturaleza debe conservarse como materia prima y que constituye un factor determinante para la sustentabilidad de la empresa (52 y 59\%, respectivamente). Tres cuartas partes piensan que la igualdad de oportunidades y los códigos de conducta favorecen la sustentabilidad, además el $76 \%$ opina que la corrupción va en contra de la sustentabilidad de la empresa. El 43\% piensa que la equidad en la distribución de 
Tabla 7

Resumen de coincidencias y diferencias entre profesores y estudiantes

\begin{tabular}{|c|c|c|c|}
\hline & $\begin{array}{l}\text { Coincidencia entre profesores } \\
\text { y estudiantes }\end{array}$ & $\begin{array}{l}\text { Percepción diferenciada } \\
\text { de los profesores }\end{array}$ & $\begin{array}{l}\text { Percepción diferenciada } \\
\text { de los estudiantes }\end{array}$ \\
\hline $\begin{array}{l}\text { Aspectos de una visión } \\
\text { integral de la } \\
\text { sustentabilidad }\end{array}$ & $\begin{array}{l}\text { La sustentabilidad se } \\
\text { conseguirá con: } \\
\text { - Equidad económica } \\
\text { - Educación } \\
\text { - Igualdad de oportunidades } \\
\text { - Establecimiento de un } \\
\text { código de conducta }\end{array}$ & $\begin{array}{l}\text { La sustentabilidad se } \\
\text { conseguirá con: } \\
\text { - Igualdad de oportunidades } \\
\text { - Principios éticos y morales } \\
\text { - Los problemas de la } \\
\text { sustentabilidad se originan } \\
\text { en: } \\
\text { - El modelo económico } \\
\text { actual } \\
\text { - Actos de corrupción } \\
\text { Están conscientes en lo } \\
\text { personal del cuidado de los } \\
\text { recursos y de la importancia } \\
\text { de conocer los antecedentes } \\
\text { del desarrollo económico }\end{array}$ & $\begin{array}{l}\text { La sustentabilidad: } \\
\text { - No solo es problema del } \\
\text { medio ambiente } \\
\text { - No solo afecta a los } \\
\text { países pobres } \\
\text { - No solo con esfuerzos } \\
\text { globales se resolverán los } \\
\text { problemas de ella }\end{array}$ \\
\hline $\begin{array}{l}\text { Aspectos con } \\
\text { conocimientos o } \\
\text { conciencia poco } \\
\text { desarrollados }\end{array}$ & $\begin{array}{l}\text { Se piensa de manera errónea } \\
\text { que los problemas de la } \\
\text { sustentabilidad se resolverán } \\
\text { mediante: } \\
\text { - La disminución de la } \\
\text { pobreza } \\
\text { - La ciencia y la tecnología } \\
\text { - El incremento del reciclado } \\
\text { en vez de la diminución en el } \\
\text { consumo } \\
\text { Perciben a la naturaleza como } \\
\text { materia prima aunque están } \\
\text { conscientes de que necesitan } \\
\text { conocer más sobre el medio } \\
\text { ambiente }\end{array}$ & $\begin{array}{l}\text { Se cree erróneamente que la } \\
\text { sustentabilidad es: } \\
\text { - Un problema del medio } \\
\text { ambiente } \\
\text { - La falta de ella afecta } \\
\text { principalmente a los países } \\
\text { pobres } \\
\text { - Podrá conseguirse } \\
\text { principalmente con esfuerzos } \\
\text { globales } \\
\text { - La sustentabilidad de la } \\
\text { empresa depende del medio } \\
\text { ambiente }\end{array}$ & $\begin{array}{l}\text { Menos conscientes de que } \\
\text { se relaciona con: } \\
\text { - Principios morales y } \\
\text { éticos } \\
\text { - Con la equidad social } \\
\text { Menos conscientes de que } \\
\text { su origen es: } \\
\text { - El modelo económico } \\
\text { actual } \\
\text { - Actos de corrupción } \\
\text { No están tan conscientes } \\
\text { en lo personal del cuidado } \\
\text { de recursos ni de la } \\
\text { importancia de conocer } \\
\text { los antecedentes del } \\
\text { desarrollo económico }\end{array}$ \\
\hline
\end{tabular}

Fuente: elaboración propia.

utilidades entre accionistas contribuye a la sustentabilidad. El $62 \%$ percibe que los problemas de sustentabilidad se originan en el modelo económico.

Respecto a los estudiantes. Aunque el $63 \%$ percibe a la naturaleza como materia prima imprescindible, el $43 \%$ - una proporción significativamente menor que los profesores - percibe que la sustentabilidad de la empresa depende del medio ambiente. No existen diferencias significativas con las percepciones de los profesores en cuanto a igualdad social y códigos de conducta, pero sí en que los estudiantes consideran en menor proporción (58\%) que la corrupción sea un obstáculo para la sustentabilidad de las empresas. No existe una diferencia significativa respecto a las opiniones sobre la equidad en la distribución de las utilidades como necesaria para la sustentabilidad. En contraste, una fracción significativamente menor que el grupo de profesores (el 43\%) percibe que los problemas de sustentabilidad se originan en el modelo económico.

A manera de resumen, en la tabla 7 se muestra la interpretación de los autores de este artículo sobre los resultados de la comparación de proporciones entre estudiantes y profesores a favor de la sustentabilidad, desde los tres ejes y para las tres categorías. Se identificaron los aspectos que 
los encuestados consideran favorables hacia la sustentabilidad y los que denotan conocimientos o conciencia poco desarrollados para el diseño de un programa de capacitación específico para profesores y de una estrategia de educación transversal para programas de licenciatura en escuelas de negocios.

A partir de la tabla anterior se puede observar que los profesores perciben que la sustentabilidad es un problema del medio ambiente y al sector de la población con menos recursos como el más afectado por dichos problemas. También, que la solución a estos problemas se conseguirá con esfuerzos globales. Por otra parte, no visualizan la equidad económica como fundamental para conseguir la sustentabilidad, ni perciben claramente el papel del eje económico.

Por su parte, los estudiantes no perciben al medio ambiente como el único factor que influye en los problemas de sustentabilidad, no perciben a la clase pobre como la responsable ni como la más afectada por dichos problemas. Ellos piensan que los esfuerzos locales también tendrán efectos en la solución de los problemas de la sustentabilidad, aunque, al igual que el grupo de profesores, solo una minoría percibe el rol del eje económico en la sustentabilidad.

\section{Conclusiones}

Con base en los resultados obtenidos a partir del análisis documental se puede concluir que las universidades evaluadas en esta investigación, a través de sus presentaciones institucionales, visualizan con mayor claridad la participación de las escuelas de negocios en el eje social de la sustentabilidad, a diferencia de los ejes ambiental y económico. Sin embargo, dado que se esperaba que el eje económico fuera claramente percibido como propio de las escuelas de negocios, el hecho de que no haya sido así da la pauta para establecer propuestas de acción para el diseño de programas académicos con énfasis en el eje económico de la sustentabilidad.

Al más alto nivel de generalización, los datos indican que los profesores tienen una percepción más integral de las relaciones entre los diferentes aspectos de la sustentabilidad, un hecho alentador dado que - al menos en teoría - son quienes actúan como difusores de conocimientos entre el alumnado. En la práctica universitaria, sin embargo, esa transferencia parece darse solo parcialmente, ya que en el área propia de las escuelas de administración, representado en la encuesta por el eje económico, los alumnos exhiben carencias significativas en su comprensión del rol de la vida económica para con la sustentabilidad. Este resultado coincide con la ausencia del eje económico en muchos de los documentos oficiales de las instituciones educativas. Definitivamente, se requiere una revisión a fondo de los planes de estudios para llevar a los estudiantes a una comprensión de la sustentabilidad como un quehacer sistémico que incluye aspectos tanto ecológicos como sociales y económicos y del rol que deben jugar las empresas específicamente en el último.

También es significativa la diferencia en la percepción entre ambos grupos sobre la responsabilidad del eje ambiental en la sustentabilidad. Los estudiantes están más conscientes de que la sustentabilidad no depende principalmente del eje ambiental. Por otra parte, también se muestran más a favor de la aseveración de que la pobreza es una consecuencia, mas no una causa, de los problemas relacionados con la sustentabilidad y que los esfuerzos locales serán más efectivos que los esfuerzos globales para conseguir el cambio. Sería aventurado afirmar que estas diferencias sean el resultado de los esfuerzos en educación para el desarrollo sustentable realizados en la educación básica entre 1995 al 2005; sin embargo, es una hipótesis que habría que validar mediante un estudio más detallado.

Cabe recordar que los resultados presentados en este texto se basan en una muestra no aleatoria de las escuelas de negocios mexicanas, donde además podría existir un sesgo de autoselección 
en el sentido de que participaron voluntariamente aquellas instituciones que de por sí perciben la necesidad de fortalecer su educación hacia la sustentabilidad, mientras que otras que no comparten esa preocupación no están representadas. No obstante lo anterior, creemos que una fortaleza de este estudio es que recoge los datos de universidades tanto públicas como privadas de diferentes zonas de la República Mexicana que voluntariamente forman parte del grupo de colaboración virtual CUDI, de forma que reflejan la diversidad de las instituciones de educación superior en el contexto nacional.

En un futuro sería deseable extender el estudio en dos dimensiones: por una parte, convendría tener muestras más grandes y/o más representativas de las universidades que podrían llevar a la formulación de recomendaciones más concretas, así como una medición más general del grado en que se incluye en la educación universitaria, la vertiente de sustentabilidad. Por otra parte, habría que revisar los programas analíticos y las herramientas didácticas específicas que se usan en diferentes instituciones para comparar su efectividad educativa, en aras de publicar un inventario de mejores prácticas en la educación.

El análisis anterior es en suma relevante y rico de información para realizar propuestas específicas, tanto para programas de capacitación docente como para el diseño de estrategias de educación transversal para programas académicos a nivel profesional en escuelas de negocios. Para complementar la información obtenida, este estudio podría enriquecerse con un análisis más detallado de los datos de percepción obtenidos, así como con otras herramientas de análisis tales como entrevistas a profundidad.

\section{Referencias}

Alonso-Almeida, M., Rodríguez, M., Cortez, K. y Abreu, J. (2012). La responsabilidad social corporativa y el desempeño financiero: un análisis en empresas mexicanas que cotizan en la bolsa. Contaduría y Administración, 57(1), 53-77.

Alcaraz, J. y Thiruvattal, E. (2010). An interview with Manuel Escudero: The United Nations' Principles for Responsible Management Education: A global call for sustainability. Academy of Management Learning and Education, 3(9), $532-541$.

Amat, F. y Moliner, L. (2010). El aprendizaje servicio en la universidad: una estrategia en la formación de ciudadanía crítica. Revista Electrónica Interuniversitaria de Formación del Profesorado, 13(4), 69-77. Disponible en: http://www.redalyc.org/articulo.oa?id=217015570006

ANFECA, Asociación Nacional de Facultades y Escuelas de Contaduría y Administración (2007). Innovación educativa en las escuelas de negocios. Ponencia central XLVIII Asamblea Nacional ANFECA, México. Disponible en: http://www.anfeca.unam.mx

Bazerman, M. H. y Tenbrunsel, A. E. (2011). Blind spots: Why we fail to do what's right and what to do about it. Princeton, NJ: Princeton University Press.

Benn, S. y Martin, A. (2010). Learning and change for sustainability reconsidered: A role for boundary objects. Academy of Management Learning and Education, 3(9), 397-412. http://dx.doi.org/10.5465/AMLE.2010.53791823

CECADESU, Centro de Educación y Capacitación para el Desarrollo Sustentable (2012). Memoria Documental «Educación y Cultura Ambiental». Disponible en: http://www.semarnat.mx/archivosanteriores/transparencia/ Documents/MEMORIA\%20CECADESU\%20FINAL\%20011112.pdf

CONAGUA, Comisión Nacional del Agua, CAEV. Comisión del Agua del Estado de Veracruz (s.f.). Encuesta para evaluar la percepción ambiental. Disponible en: http://www.cuencacoatza.org/dld/oficiales/Diagnostico_ EAS\%20_v04_distribuido.pdf

Donaldson, L. (2002). Damned by our own theories: Contradictions between theories and management education. Academy of Management Learning and Education, 1(1), 96-106. http://dx.doi.org/10.5465/AMLE.2002.7373701

Farías Martínez, G. M. y Lavín-Verástegui, J. (2012). Perfil y prácticas educativas del docente orientado a la innovación en las escuelas de negocios en México. Revista Iberoamericana de Educación Superior, 3(6), 117-127. Disponible en: https://ries.universia.net/article/view/76/perfil-practicas-educativas-docente-orientado-innovacion-escuelas-negociosmexico 
Fisher, D., Sax, C. y Grove, K. A. (2000). The resilience of changes promoting inclusiveness in an urban elementary school. Elementary School Journal, 100(3), 213-227. http://dx.doi.org/10.1086/499640

Garner, J. y Stead, W. (2010). Sustainability comes to management education and research: A story of coevolution. Academy of Management Learning and Education, 3(9), 488-498.

Gordon, I. (1998). Enhancing students' knowledge of social responsibility accounting. Issues in Accounting Education, 13(1), 31-46.

Gros, B. y Contreras, D. (2006). La alfabetización digital y el desarrollo de competencias ciudadanas. Revista Iberoamericana de Educación, (42), 103-125. Disponible en: http://www.rieoei.org/rie42a06.pdf

Haugh, H. y Talwar, A. (2010). How do corporations embed sustainability across the organization? Academy of Management Learning and Education, 3(9), 384-396. http://dx.doi.org/10.5465/AMLE.2010.53791822

Heuer, M. (2010). Foundations and Capstone; Core Values and Hot Topics; Ethics-LX; SkyTech; and The Green Business Laboratory: Simulations for sustainability education. Academy of Management Learning and Education, 3(9), 556-560. http://dx.doi.org/10.5465/AMLE.2010.53791837

Kerlinger, F. N. y Lee, H. B. (2002). Investigación del comportamiento: métodos de investigación en ciencias sociales. México: McGraw-Hill Interamericana.

Krippendorff, K. (1997). Metodología de análisis de contenido: teoría y práctica. Barcelona: Paidós.

López Noguero, F. (2002). El análisis de contenido como método de investigación. Revista de Educación, (4), 167-179.

Meira, P. (2006). Crisis ambiental y globalización: una lectura para educadores ambientales en un mundo insostenible. Trayectorias, 8(20-21), 110-123.

Morín, E. (1999). Siete saberes para la educación del futuro. París: UNESCO.

Murga-Menoyo, A. (2008). Percepciones, valores y aptitudes ante el desarrollo sostenible. Detección de necesidades educativas en estudiantes universitarios. Revista Española de Pedagogía, 240, 327-344.

Novo, M. (2009). La educación ambiental, una genuina educación para el desarrollo sostenible. Madrid: Universidad Nacional de Educación a Distancia (UNED). Cátedra UNESCO de Educación Ambiental y Desarrollo Sostenible.

OCDE, Organización para la Cooperación y el Desarrollo Económicos (2008). Desarrollo sostenible: vincular la economía, la sociedad, el medio ambiente. Disponible en: http:/www.oecd.org/insights/41774475.pdf

OIT, Organización Internacional del Trabajo (2016). Trabajo decente en América Latina y el Caribe. Disponible en: http://www.ilo.org/americas/trabajo-decente-america-latina-caribe/lang-es/index.htm

Quiroz, M. R. y Sierra, A. B. (2008). Pertinencia de las ANP como política de conservación y mejoramiento de la calidad de vida. Análisis de percepción en la Reserva de la Biosfera del Alto Golfo de California y Delta del Río Colorado. Estudios Sociales: Revista de Investigación Científica, 16(32), 141-176.

Reyes, F. y Bravo, M. (2008). Educación ambiental para la sustentabilidad en México: Aproximaciones conceptuales, metodológicas y prácticas. Tuxtla Gutiérrez, Chiapas, México: Universidad de Ciencias y Artes de Chiapas.

Rillo, A. G. (2015). Análisis hermenéutico de la relación entre desarrollo sostenible y competencias profesionales del médico general mexicano. Foro de Educación, 13(19), 263-293. Disponible en: http://dx.doi.org/10.14516/ fde.2015.013.019.012

Sandrea, L. y Reyes, L. M. (2010). Proyectos de aprendizaje de servicio comunitario y su influencia en las conductas prosociales de estudiantes universitarios. Revista de Pedagogía, 31(81), 379-401. Disponible en: http://redalyc.uaemex.mx/redalyc/src/inicio/ArtPdfRed.jsp?iCve=65919436007

SEMARNAT, Secretaría de Medio Ambiente y Recursos Naturales (2006). Estrategia nacional de educación ambiental para la sustentabilidad en México. Disponible en: http://www2.inecc.gob.mx/publicaciones/download/ 579.pdf

SEP, Secretaría de Educación Pública (2013). Programa Sectorial de Educación (2013-2018). México. Disponible en: https://www.sep.gob.mx/work/models/sep1/Resource/4479/4/images/PROGRAMA_SECTORIAL_DE_ EDUCACION_2013_2018_WEB.pdf

Shrivastava, P. (2010). Pedagogy of passion for sustainability. Academy of Management Learning and Education, 9(3), 443-455. http://dx.doi.org/10.5465/AMLE.2010.53791826

Tavakol, M. y Dennick, R. (2011). Making sense of Cronbach's alpha. International Journal of Medical Education, 2 , 53-55. http://dx.doi.org/10.5116/ijme.4dfb.8dfd

UN, United Nations (s.f.). Objetivos de Desarrollo Sostenible. Disponible en: http://www.un.org/sustainabledevelopment/ es/

UNESCO, United Nations Educational, Scientific and Cultural Organization (s.f.,a). Educación para el desarrollo sostenible. Disponible en: http://www.unesco.org/new/es/our-priorities/sustainable-development

UNESCO. United Nations Educational, Scientific and Cultural Organization (s.f.,b). La educación encierra un tesoro. Informe a la UNESCO de la Comisión internacional sobre educación para el siglo XXI, presidida por Jacques Delors. Disponible en: http://www.unesco.org/education/pdf/DELORS_S.PDF 
UNESCO, United Nations Educational, Scientific and Cultural Organization (2014). Aichi Nagoya Declaration on Education for Sustainable Development. Disponible en: https://sustainabledevelopment.un.org/content/ documents/5859Aichi-Nagoya_Declaration_EN.pdf

UNESCO, United Nations Educational, Scientific and Cultural Organization (2015). Educación 2030: Declaración de Incheon y Marco de Acción hacia una educación inclusiva y equitativa de calidad y un aprendizaje a lo largo de la vida para todos. Disponible en: http://portal.mec.gov.br/index.php?option=com_docman\&view=download\&alias= 25981-spanish-version-ffa-and-ffa-and-incheon-declaration-1 nov-2015-pdf\&Itemid=30192

UNGC, United Nations Global Compact (2009). Civismo empresarial en la economía mundial. Disponible en: https://www.unido.org/fileadmin/media/documents/pdf/Procurement/Global_Compact/GC_Brochure_Spanish.PDF

UNGC, United Nations Global Compact (2013). The smartest investment: A framework for business engagement in education. Disponible en: https://www.unglobalcompact.org/docs/issues_doc/development/Business_ Education_Framework.pdf

Wu, Y.-C. J., Huang, S., Kuo, L. y Wu, W.-H. (2010). Management education for sustainability: A web-based content analysis. Academy of Management Learning and Education, 3(9), 520-531. http://dx.doi.org/10.1108/IJSHE-10-2013-0136 